\title{
Permeability imaging in cerebrovascular diseases: applications and progress in research
}

\author{
Hui Chen, Nan Liu, Ying Li, Fei Chen and Guangming Zhu*
}

\begin{abstract}
Cerebrovascular disease is currently the second most common cause of death after ischemic heart disease. In this article, we mainly focus on the application of permeability imaging in cases of ischemic and hemorrhagic stroke, and cerebral small vessel disease. In this review, we discuss the application of permeability imaging in ischemic stroke from two aspects: 1) for the prediction of hemorrhagic transformation after infarction, and 2) for the evaluation of newborn secondary and tertiary collateral circulations. Quantitative measurements of blood-brain barrier (BBB) disruption by the Dynamic Contrast Enhance MR (DCE-MR) can reveal the severity of intracranial hemorrhage (ICH)-induced brain damage, and that the technique has the potential to be used for testing the efficacy of interventions aimed at reducing tissue damage around the hematoma. Currently, DCE-MR is mostly applied for the assessment of tumors in patients. There is less research focused on the evaluation of mild BBB defects in normal or abnormal aging brains, dementia, or cerebral small vessel disease. More work needs to be done to select the appropriate contrast agents and decide their doses, as well as to identify methods for parameter collection and data analysis.
\end{abstract}

Keywords: Permeability imaging, Blood-brain barrier, $K^{\text {trans }}$, Dynamic Contrast Enhance MR, ischemic stroke, Hemorrhagic transformation, Collateral circulation, Hemorrhagic stroke, Cerebral small vessel disease

\section{Background}

Cerebrovascular disease is currently the second most common cause of death after ischemic heart disease, and a leading cause of disability [1]. Every year, 15 million people worldwide experience ischemic or hemorrhagic stroke [2]. Modern imaging techniques not only clarify the nature of the stroke within minutes, but also play critical roles in identifying the cause of the stroke, guiding the late-stage treatment, and evaluating the prognosis [2]. Large numbers of molecular imaging techniques, including permeability imaging, have gradually entered clinical practice, and help physicians who are involved in neurosurgery and neuroimaging, gain a deeper understanding of the pathophysiological changes in nervous system disorders.

Permeability imaging is often used in the diagnosis and prognosis of brain tumors, as well as in the blood-

\footnotetext{
* Correspondence: zhugmdc@aliyun.com

Department of Neurology, Military General Hospital of Beijing PLA, Beijing 100700, China
}

(c) 2016 Chen et al. Open Access This article is distributed under the terms of the Creative Commons Attribution 4.0 International License (http://creativecommons.org/licenses/by/4.0/), which permits unrestricted use, distribution, and reproduction in any medium, provided you give appropriate credit to the original author(s) and the source, provide a link to the Creative Commons license, and indicate if changes were made. The Creative Commons Public Domain Dedication waiver (http://creativecommons.org/publicdomain/zero/1.0/) applies to the data made available in this article, unless otherwise stated. brain barrier permeability (BBBP) assessment for ischemic cerebrovascular disease, spontaneous intracerebral hemorrhage $(\mathrm{ICH})$, cerebral small vessel disease, cognitive dysfunction, multiple sclerosis, and brain trauma [3-6]. In this article, we mainly focus on the application of permeability imaging in cases of ischemic and hemorrhagic stroke, and cerebral small vessel disease.

\section{Review}

Neurovascular unit, BBB, and BBB permeability

The process of tissue damage after stroke is highly complex, involving changes in brain vasculature and parenchyma that are regulated by the interactions of a variety of mechanisms. More and more studies suggest that the treatment of cerebrovascular diseases must go beyond the concept of cell damage alone. It is important to pay more attention to the dynamic changes in the neurovascular units (NVU), which are the integrative microunits of structure and function of the nervous system. Thus, neurons, endothelial cells, astrocytes, and the extracellular 
matrix that maintains the integrity of the brain tissue need to be viewed comprehensively, with the BBB acting as the core of the NVU [7]. The negative results from clinical trials of neuro-protective drugs also support this idea, making the NVU an important therapeutic target in future clinical research. The extent of damage and the late-stage recovery of the NVU determine the clinical outcome of patients. However, no ideal method can assess NVU dysfunction accurately or quantitatively.

Some researchers quantify the damage to the NVU by examining specific markers, such as Matrix Metalloproteinases (MMPs), Vascular Endothelial Growth Factor (VEGF), Platelet Derived Growth Factor (PDGF), and Fibroblast Growth Factor (FGF), in blood or cerebrospinal fluid [8]. However, the specificity of this method is low, spatially, as it cannot distinguish the location and the extent of NVU damage in different brain regions. Double-photon laser-scanning microscopy, which can detect the dynamic relationship between the microvasculature and the surrounding structure in living tissue, is the ideal imaging method for examining the NVU. However, due to the complicated scanning procedure involved and the need for a craniotomy in order to expose the areas of interest, Double-photon microscopy is currently used only in animal models [9].

The BBB, which is composed of capillary endothelial cells, basement membrane, pericytes located outside the basement membrane, and the perivascular end-feet of astrocytes, is the most important protective structure in the brain. It can reduce the passive movement of water molecules and restrict the passage of soluble substances from the blood, thereby preventing brain cells from being exposed to neurotoxins or other harmful bloodborne substances. The BBB is considered as the core structure of the NVU, and the defects in structure and function can be found in most neurological diseases.

Disorders of the BBB are particularly evident in ischemic cerebrovascular disease, and show dynamic changes under different states of tissue damage and reperfusion. After ischemic brain damage, BBB leakage occurs not only during the acute and subacute phases of stroke [10], but also at the early stage of angiogenesis during stroke recovery [11]. In addition, studies have found that BBB disruption is most significant at the edges of the hematoma, 1 week after spontaneous $\mathrm{ICH}$, and quantitative measurement of the $\mathrm{BBB}$ damage can reflect the extent of $\mathrm{ICH}$-induced brain damage. Damage to the BBB can also be found in the early stage of lacunar infarction, white matter osteoporosis, and other cerebral small vessel diseases [12].

The severity of $\mathrm{BBB}$ damage is positively correlated with the degree of brain tissue damage or hypoxia and ischemia, and therefore quantitative assessment of the disruption of the BBB (i.e., the BBBP) can be used for quantitative evaluation of the severity of the NVU damage [13].

\section{Principles of permeability imaging}

Permeability imaging uses classic pharmacokinetic theory to quantitatively assess the rate at which a contrast agent passes through the BBB [14]. The increase in $\mathrm{BBBP}$ reflects BBB-relevant pathophysiological changes, and therefore the quantitative description of the BBBP has important clinical significance [15]. Common parameters include volume transfer constant $\left(\mathrm{K}^{\text {trans }}\right)$ and permeability-surface area product (PS). Between the two, $\mathrm{K}^{\text {trans }}$ is generally believed to represent permeability.

Though there are multiple methods to obtain permeability parameters, such as first pass data of perfusion CT and Dynamic Susceptibility Contrast MR, the standard method for BBB permeability assessments are based on Dynamic contrast-enhanced MR (DCE-MR). The DCE-MR scanning process begins with multiple flipangle $\mathrm{T} 1$ sequences, followed by intravenous injection of contrast agent, after which the T1-weighted GRE sequence is acquired over several minutes. The observation of a linear relationship between the MR signal intensity and the scan time indicates that the slope is associated with BBB permeability [2]. In patients with subsequent hemorrhagic transformation (HT), even an enhanced T1 sequence does not exhibit a visually identifiable enhanced effect. However, the increase in BBB permeability at this stage can be observed in DCE-MR [16]. Studies have indicated that the DCE acquisition time should be at least $210 \mathrm{~s}$ [17] in order to distinguish between patients with HT from those without HT.

Currently, the tracer kinetics model used in most permeability imaging is the corrected single-capillary model proposed by Larsson and Tofts [18, 19]. However, this model requires hemodynamic balance, which calls for relatively long scan times. The Patlak model [20] only analyzes the first-pass data of the contrast agent. Requiring lesser amounts of data, it has been successfully used to analyze permeability data and obtain relevant parameters such as $K^{\text {trans }}$. Multiple studies using Patlak data analysis have correctly assessed the permeability parameter $K^{\text {trans }}$ in stroke patients [21]. However, this approach can only be used in cases with moderate BBB leakage. When there is severe BBB leakage, data collection takes a longer time and the results are biased.

In the future, quantitative assessment of BBB leakage needs to mainly focus on reducing errors, particularly on optimizing the assessment of arterial input function to reduce errors caused by different tracers.

\section{Application in ischemic brain injury}

The defect of BBB occurs rapidly after acute cerebral infarction and is accompanied by a significant increase in 
BBBP. Studies have shown that the average time to BBB defect after the onset of cerebral ischemia is $3.8 \mathrm{~h}$, which is similar to the time at which irreversible brain damage occurs [13].

Here, we discuss the application of permeability imaging in two aspects: 1) for the prediction of HT after infarction, and 2) for the evaluation of newborn secondary and tertiary collateral circulations.

Symptomatic HT is one of the most serious complications of acute ischemic stroke and is closely related to clinical outcomes [22]. Currently, the commonly used imaging techniques cannot directly assess the risk of HT. A number of studies have demonstrated that enhanced MR is highly capable of predicting HT [23, 24]. However, the methods described above can only provide indirect evidence of increased permeability resulting from BBB disruption. They cannot provide quantification data and require highly experienced evaluators for correct interpretation [23]. Using the DCE-MR to quantitatively evaluate the $\mathrm{BBB}$, the $\mathrm{K}^{\text {trans }}$ has proved to be the most sensitive imaging marker for the prediction of early (within 2-3 h) fibrin leakage in the brain tissue [24]. Kassner et al. added the DCE sequence in the conventional MR of 33 patients within $4 \mathrm{~h}$ of the onset of acute cerebral infarction and found progressive increase in the BBBP in the acute phase of nine patients (five patients received tPA thrombolytic therapy), all of whom presented with HT within $48 \mathrm{~h}$ [25]. However, due to the long scan time of the DCE-MR, its application in the ultra-early stage of acute cerebral infarction is limited. Thus, some studies obtained BBBP values from first-pass perfusion CT (PCT) data. For example, Wintermark and Lee et al. applied different mathematical models to measure the absolute values of BBBP and proposed that the increase in the BBBP can be utilized to predict HT [26]. Permeability imaging can systematically assess BBB integrity and make personalized predictions regarding the risk of hemorrhage in patients with acute ischemic stroke, which should help realize the transformation from the "time-window" to the "tissue-window" approach. Thus, patients who have a high risk of HT with active treatments even within the 4.5-h therapeutic time window, as well as those who have a relatively low risk of HT and relatively good prognosis even beyond the 4.5-h window, can be screened out. That is, it will be possible to move away from the fixed time-window treatment model and develop more rational therapies based on the evaluation of individualized risks [27].

The intracranial collateral circulation plays a critical role in the occurrence, development, treatment, and prognosis of ischemic stroke. The collateral circulation is capable of maintaining perfusion and stabilizing cerebral blood flow, which in turn, determines the tissue outcomes. Studies have reported that, in the patients with severe carotid artery stenosis, a good collateral circulation could reduce the incidence of long-term stroke, perioperative risk, and transient ischemic attack [28, 29]. Regardless of the success of recanalization of occluded vessels after thrombolytic therapy, the long-term prognosis of patients with leptomeningeal collateral vessels is better than that of other patients [30].

Methods for assessing intracranial collaterals include transcranial Doppler (TCD) ultrasound, computed tomography angiography (CTA), MR angiography (MRA), and digital subtraction angiography (DSA), each of which has its own advantages and disadvantages. For the assessment of secondary collaterals, permeability imaging has sufficient theoretical basis. The dynamic contrast-enhanced sequence itself contains perfusion information. In a study on the assessment of the collateral circulation in patients with acute stroke, Chen et al. [30] used first-pass data of perfusion images to obtain the permeability parameter $\mathrm{K}^{\text {trans }}$ map, and found that the $\mathrm{K}^{\text {trans }}$ map can assess collateral circulation in the acute ischemic state. The corresponding collateral circulation score is most consistent with that of DSA. Chen et al. also found that the $\mathrm{K}^{\text {trans }}$ map can predict clinical outcomes after stroke.

$\mathrm{K}^{\text {trans }}$, as well as measurements from other sequences in MR, also confirmed the location and the size of the area of revascularization and angiogenesis [31]. Similar to its usefulness in detecting tumor angiogenesis, $\mathrm{K}^{\text {trans }}$ is a sensitive parameter for the detection of early brain angiogenesis in post-stroke patients. Although previous research on the detection of angiogenesis by DCE-MR had only revealed that increased intensity of the $\mathrm{K}^{\text {trans }}$ signal corresponds to increased density of newborn vessels, it was not clear whether secondary and tertiary cerebral collaterals could be evaluated through the assessment of angiogenesis. In a recent study, Chen et al. [32] collected 21 patients with severe intracranial arterial stenosis or occlusion caused by chronic artery atherosclerosis. The patients all presented with severe stenosis or occlusion of the middle cerebral artery and the intracranial segment of the internal carotid artery. The study used the corrected Alberta Stroke Program Early CT Score (ASPECTS) segmentation standard to evaluate collateral circulations in each of the vasculature segments and used $K^{\text {trans }}$ maps, Arterial Spin Labeling (ASL), CTA, and DSA to score collaterals. The authors found good agreement between the DSA and $\mathrm{K}^{\text {trans }}$ map, especially in the assessment of the meningeal collateral circulation. The agreement between the CTA-source image (CTA-SI) and DSA was moderate, while the agreement between the ASL and DSA was the least favorable. However, the sample size in this study was too small to draw definite conclusions.

Although $\mathrm{K}^{\text {trans }}$ has been applied in cancer patients [33], it is rarely used in stroke patients. This may be due 
to the lack of awareness regarding the usefulness of $\mathrm{K}^{\text {trans }}$ measurements. Between perfusion imaging and permeability imaging, physicians usually prefer the former. In fact, permeability imaging itself already contains perfusion information. With optimization of the software, a good perfusion sequence can also be obtained during permeability imaging.

\section{Application in hemorrhagic stroke}

Hypertensive $\mathrm{ICH}$ is the deadliest and most disabling form of stroke and affects nearly a million people worldwide each year [34]. Studies using animal models have shown that the toxic effects of hemoglobin degradation products can cause increases in the BBB permeability and lead to the formation of edema around the hematoma [35]. Studies have speculated that disruption of the $\mathrm{BBB}$ can lead to angiogenesis in the vicinity of the hematoma, which further promotes the formation of vasogenic edema [36]. Therefore, BBB disruption may be an important pathophysiological factor involved in hypertensive $\mathrm{ICH}$-induced brain damage, and is a potential target for therapeutic intervention [35].

The study by Didem et al. showed that the DCE-MR could demonstrate an increase in BBB permeability in the boundary region of the hematoma 8 days after cerebral hemorrhage. However, no contrast agent was found in the hematoma itself, and BBBP was not increased in the contralateral hemisphere [37]. Research on spontaneous ICH in humans has shown that contrast enhancement can be observed around the hematoma in $60 \%$ of patients 5 days after the occurrence of the ICH [38]. No contrast enhancement can be found in the hematoma itself, possibly due to blood clots preventing the leakage of contrast agents. In an ICH rat model, Yang et al. observed increased BBB permeability both in the core and at the edge of the hematoma 7 days after the $\mathrm{ICH}$ [36]. However, their ICH model was created by direct injection of autologous blood instead of blood vessel rupture and can therefore not reflect the real pathophysiological changes of ICH in humans.

A study by Didem et al. revealed the relationship between $\mathrm{BBB}$ leakage and the size of the hematoma using DCE-MR. BBB leakage is more severe around large hematomas (i.e., $\geq 30 \mathrm{~mL}$ ), and a higher increase in the BBBP, as well as its variability, occurred more often in larger hematomas than in smaller ones [37]. This is consistent with the observation that edema volume in bigger hematomas is greater than that in smaller hematomas [39]. BBB leakage varies depending on hemorrhage location. Regardless of the size of the hematoma, BBB permeability and variability is higher in lobar than in deep hemorrhages [37].

Animal studies have indicated that the BBB permeability starts to increase only hours after $\mathrm{ICH}$, and continues until 48-72 $\mathrm{h}$ [36]. In these models, the amount of BBB leakage gradually declined after the peak, which occurred in the first few days after the onset of ICH. However, the increase in BBBP was sustained for up to 14 days [36]. One animal study found that the measurements of the BBBP were similar between 1 week and 1day post-ICH [40].

Taken together, the evidence suggests that quantitative measurements of BBB disruption by the DCE-MR can reveal the severity of $\mathrm{ICH}$-induced brain damage, and that the technique has the potential to be used for testing the efficacy of interventions aimed at reducing tissue damage around the hematoma [37].

\section{Application in cerebral small vessel disease}

Cerebral small vessel disease can cause dementia and stroke. The most characteristic imaging manifestations include lacunar infarction [41], leukoaraiosis, enlarged perivascular spaces, and cerebral microbleeds [42]. Studies have demonstrated that endothelial injuries can cause BBB leakage at multiple sites, which leads to ongoing damage of the vessel wall and eventually to blood vessel ruptures and microbleeds [43]. These microbleeds together with reactive small-vessel occlusions induce cystic infarcts of the surrounding parenchyma. Schreiber et al. reported that in spontaneously hypertensive strokeprone rats, the vascular system reacts with an activated coagulation state after the early endothelial injuries and induces stasis formation and the accumulation of erythrocytes, which represent the earliest detectable histological characteristics of small vessel disease [43].

Many studies have reported that increased BBB permeability occurs in the aging brain, dementia, and leukoaraiosis in humans. However, it must be noted that the sample size of the study on leukoaraiosis was small and the results are unreliable [44]. Most of the studies examined the BBBP using biochemical methods, such as measurement of the cerebrospinal fluid (CSF) albumin/serum albumin ratio. Several studies have used imaging techniques to examine the $\mathrm{BBB}$, mostly through intravenous injection of the MR contrast agent gadolinium, a relatively nonspecific marker for detection of the BBB disruption [44]. Topakian et al. studied 24 patients with lacunar infarction and compared them with controls. They found that in leukoaraiosis patients, DCE-MR revealed increased BBBP even in regions of the white matter that appeared normal [3]. Rosenberg et al. studied patients with vascular cognitive impairment and confirmed the disruption of the BBB in the areas of leukoaraiosis [45].

Overall, BBB integrity deteriorates slowly with aging, with the decline being more severe in patients with dementia and small vessel disease. BBB damage plays an important role in lacunar infarction, leukoaraiosis, other 
brain small vessel diseases, and age-related diseases (such as Alzheimer disease). Preliminary reports suggest that the BBB defect is present even before the clinical and imaging manifestations arise. The enlarged perivascular space is an important marker for cerebral small vessel disease, and brain damage caused by inflammation and other pathological processes is a marker for the initial damage to the BBB [12].

Long-term follow-up studies are required to determine the role of $\mathrm{BBB}$ damage in the pathology of cerebral small vessel disease. The BBB can be quantitatively evaluated by DCE-MR, which can be combined with pathology methods to identify the major mechanisms of BBB damage and further explore its pathogenesis. Currently, DCE-MR is mostly applied for the assessment of tumors in patients. There is less research focused on the evaluation of mild BBB defects in normal or abnormal aging brains, dementia, or cerebral small vessel disease. More work needs to be done to select the appropriate contrast agents and decide their doses, as well as to identify methods for parameter collection and data analysis [46].

\section{Conclusions}

This review discusses the applications of DCE-MR-based permeability imaging techniques in cerebrovascular diseases. With regard to the methods, T1-weighted DCEMR is more developed. However, due to the lack of a unified standard for the image acquisition, data models, and study reports, it is difficult to compare and analyze DCE data between different studies. Further improvements for enhancing the reliability and stability of the DCE-MR are needed for its application in the assessment of subtle changes in the permeability of the BBB. Future research should attempt to establish a unified data collection and analysis method, which should help improve the comparability between studies and promote the wide application of DCE-MR in clinical practice and research [46].

\section{Abbreviations \\ ASL: Arterial Spin Labeling; BBB: Blood-Brain Barrier; BBBP: blood-brain barrier permeability; CTA: computed tomography angiography; CTA: SI-CTA source image; DCE: Dynamic Contrast Enhance; DSA: digital subtraction angiography; FGF: Fibroblast Growth Factor; HT: hemorrhagic transformation; ICH: intracranial hemorrhage; MMPs: Matrix MetalloProteinases; MRA: MR angiography; NVU: neurovascular units; PDGF: Platelet Derived Growth Factor; PS: surface area product; PCT: perfusion CT; TCD: transcranial Doppler; VEGF: Vascular Endothelial Growth Factor.}

\section{Competing interests}

The authors declare that they have no competing interests.

\section{Authors' contributions}

$\mathrm{HC}$ consulted literatures and wrote the review. NL, YL and FC carried out the reference collection. GZ conceived of the idea, and participated in its design and coordination and helped to draft the manuscript. All authors read and approved the final manuscript.

\section{Acknowledgements}

This review was supported by the National Natural Science Foundation of China (Grant No. 81371286 and No. 81501024).

Received: 20 November 2015 Accepted: 7 January 2016

Published online: 04 February 2016

\section{References}

1. Panchal HB, Ladia V, Amin P, Patel P, Veeranki SP, Albalbissi K, et al. A metaanalysis of mortality and major adverse cardiovascular and cerebrovascular events in patients undergoing transfemoral versus transapical transcatheter aortic valve implantation using edwards valve for severe aortic stenosis. Am J Cardiol. 2014;114:1882-90.

2. Hoffmann A, Zhu G, Wintermark M. Advanced neuroimaging in stroke patients: prediction of tissue fate and hemorrhagic transformation. Expert Rev Cardiovasc Ther. 2012;10:515-24

3. Topakian R, Barrick TR, Howe FA, Markus HS. Blood-brain barrier permeability is increased in normal-appearing white matter in patients with lacunar stroke and leucoaraiosis. J Neurol Neurosurg Psychiatry. 2010;81: 192-7.

4. van de Haar HJ, Burgmans S, Hofman PA, Verhey FR, Jansen JF, Backes WH. Blood-brain barrier impairment in dementia: current and future in vivo assessments. Neurosci Biobehav Rev. 2015;49:71-81.

5. Alluri $\mathrm{H}$, Wiggins-Dohlvik K, Davis ML, Huang JH, Tharakan B. Blood-brain barrier dysfunction following traumatic brain injury. Metab Brain Dis. 2015; 30:1093-104.

6. Cramer SP, Larsson HB. Accurate determination of blood-brain barrier permeability using dynamic contrast-enhanced T1-weighted MRI: a simulation and in vivo study on healthy subjects and multiple sclerosis patients. J Cereb Blood Flow Metab. 2014;34:1655-65.

7. Muoio V, Persson PB, Sendeski MM. The neurovascular unit - concept review. Acta Physiol (Oxf). 2014;210:790-8.

8. Amtul Z, Hepburn JD. Protein markers of cerebrovascular disruption of neurovascular unit: immunohistochemical and imaging approaches. Rev Neurosci. 2014;25:481-507.

9. Tran $\mathrm{CH}$, Gordon GR. Acute two-photon imaging of the neurovascular unit in the cortex of active mice. Front Cell Neurosci. 2015;9:11.

10. Leigh R, Jen SS, Hillis AE, Krakauer JW, Barker PB, Stir, et al. Pretreatment blood-brain barrier damage and post-treatment intracranial hemorrhage in patients receiving intravenous tissue-type plasminogen activator. Stroke. 2014:45:2030-5.

11. Sun FL, Wang W, Cheng H, Wang Y, Li L, Xue JL, et al. Morroniside improves microvascular functional integrity of the neurovascular unit after cerebral ischemia. PLoS One. 2014;9, e101194.

12. Wardlaw JM. Blood-brain barrier and cerebral small vessel disease. J Neurol Sci. 2010;299:66-71.

13. Chassidim Y, Vazana U, Prager O, Veksler R, Bar-Klein G, Schoknecht K, et al. Analyzing the blood-brain barrier: the benefits of medical imaging in research and clinical practice. Semin Cell Dev Biol. 2015;38:43-52.

14. Tofts PS, Brix G, Buckley DL, Evelhoch JL, Henderson E, Knopp MV, et al. Estimating kinetic parameters from dynamic contrast-enhanced T(1)weighted MRI of a diffusable tracer: standardized quantities and symbols. J Magn Reson Imaging. 1999;10:223-32.

15. Nagaraja TN, Keenan KA, Aryal MP, Ewing JR, Gopinath S, Nadig VS, et al. Extravasation into brain and subsequent spread beyond the ischemic core of a magnetic resonance contrast agent following a step-down infusion protocol in acute cerebral ischemia. Fluids Barriers CNS. 2014;11:21.

16. Kassner A, Roberts T, Taylor K, Silver F, Mikulis D. Prediction of hemorrhage in acute ischemic stroke using permeability MR imaging. AJNR Am J Neuroradiol. 2005:26:2213-7.

17. Vidarsson L, Thornhill RE, Liu F, Mikulis DJ, Kassner A. Quantitative permeability magnetic resonance imaging in acute ischemic stroke: how long do we need to scan? Magn Reson Imaging. 2009;27:1216-22.

18. Tofts PS, Kermode AG. Measurement of the blood-brain barrier permeability and leakage space using dynamic MR imaging. 1. Fundamental concepts. Magn Reson Med. 1991;17:357-67.

19. Larsson HB, Stubgaard M, Frederiksen JL, Jensen M, Henriksen O, Paulson OB. Quantitation of blood-brain barrier defect by magnetic resonance imaging and gadolinium-DTPA in patients with multiple sclerosis and brain tumors. Magn Reson Med. 1990;16:117-31. 
20. Patlak CS, Blasberg RG. Graphical evaluation of blood-to-brain transfer constants from multiple-time uptake data. Generalizations. J Cereb Blood Flow Metab. 1985;5:584-90.

21. Nagaraja TN, Knight RA, Ewing JR, Karki K, Nagesh V, Fenstermacher JD. Multiparametric magnetic resonance imaging and repeated measurements of blood-brain barrier permeability to contrast agents. Methods Mol Biol. 2011;686:193-212.

22. Asuzu D, Nystrom K, Amin H, Schindler J, Wira C, Greer D, et al. Modest Association between the Discharge Modified Rankin Scale Score and Symptomatic Intracerebral Hemorrhage after Intravenous Thrombolysis. J Stroke Cerebrovasc Dis. 2015;24:548-53.

23. Knight RA, Barker PB, Fagan SC, Li Y, Jacobs MA, Welch KM. Prediction of impending hemorrhagic transformation in ischemic stroke using magnetic resonance imaging in rats. Stroke. 1998;29:144-51.

24. Jiang Q, Ewing JR, Ding GL, Zhang L, Zhang ZG, Li L, et al. Quantitative evaluation of BBB permeability after embolic stroke in rat using MRI. J Cereb Blood Flow Metab. 2005:25:583-92.

25. Adraktas DD, Brasic N, Furtado AD, Cheng SC, Ordovas K, Chun K, et al. Carotid atherosclerosis does not predict coronary, vertebral, or aortic atherosclerosis in patients with acute stroke symptoms. Stroke. 2010;41: 1604-9.

26. Dankbaar JW, Hom J, Schneider T, Cheng SC, Bredno J, Lau BC, et al. Dynamic perfusion-CT assessment of early changes in blood brain barrier permeability of acute ischaemic stroke patients. J Neuroradiol. 2011;38:161-6.

27. Kassner A, Mandell DM, Mikulis DJ. Measuring permeability in acute ischemic stroke. Neuroimaging Clin N Am. 2011;21:315-25. x-xi.

28. Winship IR, Armitage GA, Ramakrishnan G, Dong B, Todd KG, Shuaib A. Augmenting collateral blood flow during ischemic stroke via transient aortic occlusion. J Cereb Blood Flow Metab. 2014;34:61-71.

29. Kobayashi J, Uehara T, Toyoda K, Endo K, Ohara T, Fujinami J, et al. Clinical significance of fluid-attenuated inversion recovery vascular hyperintensities in transient ischemic attack. Stroke. 2013:44:1635-40.

30. Chen H, Wu B, Liu N, Wintermark M, Su Z, Li Y, et al. Using standard firstpass perfusion computed tomographic data to evaluate collateral flow in acute ischemic stroke. Stroke. 2015;46:961-7.

31. Li L, Jiang $Q$, Zhang $L$, Ding G, Wang $L$, Zhang R, et al. Ischemic cerebra tissue response to subventricular zone cell transplantation measured by iterative self-organizing data analysis technique algorithm. J Cereb Blood Flow Metab. 2006;26:1366-77.

32. Chen H, Wu B, Zhu G, Wintermark M, Wu X, Su Z, et al. Permeability Imaging as a Biomarker of Leptomeningeal Collateral Flow in Patients with Intracranial Arterial Stenosis. Cell Biochem Biophys. 2014

33. Franiel T, Hamm B, Hricak H. Dynamic contrast-enhanced magnetic resonance imaging and pharmacokinetic models in prostate cancer. Eur Radiol. 2011;21:616-26.

34. Roger VL, Go AS, Lloyd-Jones DM, Adams RJ, Berry JD, Brown TM, et al. Heart disease and stroke statistics-2011 update: a report from the American Heart Association. Circulation. 2011;123:e18-209.

35. Silva-Candal AD, Vieites-Prado A, Gutierrez-Fernandez M, Rey Rl, Argibay B, Mirelman D, et al. Blood glutamate grabbing does not reduce the hematoma in an intracerebral hemorrhage model but it is a safe excitotoxic treatment modality. J Cereb Blood Flow Metab. 2015

36. Yang D, Knight RA, Han Y, Karki K, Zhang J, Ding C, et al. Vascular recovery promoted by atorvastatin and simvastatin after experimental intracerebral hemorrhage: magnetic resonance imaging and histological study. J Neurosurg. 2011;114:1135-42.

37. Aksoy D, Bammer R, Mlynash M, Venkatasubramanian C, Eyngorn I, Snider RW, et al. Magnetic resonance imaging profile of blood-brain barrier injury in patients with acute intracerebral hemorrhage. J Am Heart Assoc. 2013;2, e000161.

38. Kidwell CS, Burgess R, Menon R, Warach S, Latour LL. Hyperacute injury marker (HARM) in primary hemorrhage: a distinct form of CNS barrier disruption. Neurology. 2011;77:1725-8.

39. Venkatasubramanian C, Mlynash M, Finley-Caulfield A, Eyngorn I, Kalimuthu R, Snider RW, et al. Natural history of perihematomal edema after intracerebral hemorrhage measured by serial magnetic resonance imaging. Stroke. 2011:42:73-80.

40. Wu G, Li C, Wang L, Mao Y, Hong Z. Minimally invasive procedures for evacuation of intracerebral hemorrhage reduces perihematomal glutamate content, blood-brain barrier permeability and brain edema in rabbits. Neurocrit Care. 2011;14:118-26.
41. Potter GM, Doubal FN, Jackson CA, Chappell FM, Sudlow CL, Dennis MS, et al. Counting cavitating lacunes underestimates the burden of lacunar infarction. Stroke. 2010;41:267-72.

42. Doubal FN, MacLullich AM, Ferguson KJ, Dennis MS, Wardlaw JM. Enlarged perivascular spaces on MRI are a feature of cerebral small vessel disease. Stroke. 2010:41:450-4.

43. Schreiber S, Bueche CZ, Garz C, Braun H. Blood brain barrier breakdown as the starting point of cerebral small vessel disease? - New insights from a rat model. Exp Transl Stroke Med. 2013;5:4.

44. Farrall AJ, Wardlaw JM. Blood-brain barrier: ageing and microvascular disease-systematic review and meta-analysis. Neurobiol Aging. 2009:30:337-52.

45. Rosenberg GA. Inflammation and white matter damage in vascular cognitive impairment. Stroke. 2009:40:\$20-3.

46. Heye AK, Culling RD, Valdes Hernandez Mdel C, Thrippleton MJ, Wardlaw JM. Assessment of blood-brain barrier disruption using dynamic contrastenhanced MRI. A systematic review. Neuroimage Clin. 2014;6:262-74.

\section{Submit your next manuscript to BioMed Central and we will help you at every step:}

- We accept pre-submission inquiries

- Our selector tool helps you to find the most relevant journal

- We provide round the clock customer support

- Convenient online submission

- Thorough peer review

- Inclusion in PubMed and all major indexing services

- Maximum visibility for your research

Submit your manuscript at www.biomedcentral.com/submit
) Biomed Central 